\title{
Induction of monocyte antitumor response by human cancer cells transduced with TNF-GFP fusion gene: possible implications for immunotherapy of cancer
}

\author{
Jerzy Więckiewicz, Bożenna Mytar, Rafał Szatanek, \\ Kazimierz Węglarczyk, Jarosław Baran
}

Department of Clinical Immunology, Polish-American Institute of Pediatrics, Jagiellonian University Medical College, Krakow, Poland

\begin{abstract}
This study was undertaken to determine how human pancreatic cancer (HPC-4) cells transduced with the TNF-GFP fusion gene (TLG) alter the antitumor response of human monocytes in vitro and whether they could act as an antitumor vaccine. In our model, HPC-4 cells were transduced with retroviral vector harboring TLG gene and designated as HPC- $4_{\text {TLG }}$. The TLG protein expression was confirmed by Western blot and flow cytometry analysis. Monocytes were co-cultured with transduced and control HPC-4 cells. The secretion of TNF, IL-10 and IL-12 was measured by ELISA. The cytotoxicity of monocytes against HPC-4 cells was determined by MTT test. The results show that the HPC-4 ${ }_{\text {TLG }}$ cells expressed membrane-bound, intracellular and secretory TLG protein. When cultured with HPC- $4_{\text {TLG }}$ cells, monocytes released a higher amount of TNF, but IL-10 and IL-12 secretion was inhibited. The pre-exposure of monocytes to HPC-4 ${ }_{\mathrm{TLG}}$, but not to HPC-4, cells did not decrease TNF nor increase IL-10 production, thus not leading to monocyte deactivation. Also, the antitumor cytotoxicity of monocytes stimulated with HPC- $4_{\text {TLG }}$ was not downregulated, which occurred when non-transduced HPC-4 cells were used. In conclusion, compared to parental HPC-4 cells, TLG gene transduced HPC-4 cells induced stronger antitumor response of monocytes in vitro and prevented deactivation of monocytes. (Folia Histochemica et Cytobiologica 2011, Vol. 49, No. 3, 512-520)
\end{abstract}

Key words: fusion gene, monocytes, tumor cells, tumor necrosis factor

\section{Introduction}

A serious problem with the retroviral system of gene transfer is that it is difficult to maintain a high level of cloned gene expression following transduction. Usually, several passages of transduced cells result in the outgrowth of clones that express much lower levels of the transgene coded protein. The use of the

Correspondence address: J. Więckiewicz,

Department of Clinical Immunology,

Polish-American Institute of Pediatrics,

Jagiellonian University Medical College,

Wielicka Str. 265, 30-663 Krakow, Poland;

tel.: (+ 48 12) 65824 86, fax: (+ 48 12) 65817 56;

e-mail:miwiecki@cyf-kr.edu.pl internal ribosome entry site (IRES) elements rather than internal promoters could increase the likelihood of stable expression of the cloned gene of interest. However, even the use of IRES sequence does not completely reduce the number of potential mechanisms, both at the transcriptional and post-transcriptional levels, that could affect the transgene expression [1]. Cloned gene expression also depends on retroviral DNA integration site in the host genome $[2,3]$. Cloning of the gene coding for green fluorescent protein (GFP) isolated from the jellyfish Aequorea victoria and its efficient species-independent expression is a new versatile reporter gene system $[4,5]$.

Autologous cancer cells used as vaccines to enhance antitumor activity have been explored extensively, but their efficiency has proved to be low. There- 
fore, genetically engineered tumor cells transfected with various cytokines coding genes have been used [6-8]. However, despite positive effects in murine experimental tumor models, their clinical efficiency in human cancer is marginal, with an objective response rate of 3.3\% [9]. Also, human monocytes that exert a direct cytotoxic effect on tumor cells, as mediated by radical oxygen intermediates (ROI) and tumor necrosis factor (TNF), upon arrival at the tumor site form tumor infiltrating macrophages (TIM), which become polarized to M2 macrophages, thus strongly reducing their cytotoxic activity [10]. This occurs due to the tumor cell products, including extracellular matrix components, IL-10, CSF-1 and other cytokines. In general, M2 macrophages have poor antigen presenting capacity, have an IL-12 $2^{\text {low }}$ and IL- $10^{\text {high }}$ phenotype, suppress the inflammatory response and Th1 adaptive immunity, and promote angiogenesis, tissue remodeling and tumor progression [10]. Such TIM changes may be responsible, at least in part, for the inefficiency of tumor vaccines.

We have previously observed that human monocytes stimulated with tumor cells produce TNF, IL-10 and IL-12 [11]. However, when monocytes were first pre-exposed to tumor cells, sorted out from coculture and then re-stimulated with tumor cells, they became M2 polarized cells that produced increased amounts of immunosuppressive IL-10 [11]. Therefore, we wanted to investigate whether the use of TNF gene transduced tumor cells might increase their immunostimulatory potential for monocytes and prevent their M2 polarization.

\section{Material and methods}

Reagents and antibodies. The oligonucleotides were purchased from TIB Molbiol (Poznan, Poland). Polymerase chain reaction (PCR) and enhanced avian reverse transcriptase (RT) kits, agarose (for routine use), polybrene (hexadimethrine bromide), and the antibiotics ampicillin, neomycin sulfate and tylosin tartrate were from Sigma Chemical Company (St. Louis, MO, USA). The Pwo DNA polymerase with the appropriate incubation buffer was obtained from Roche (Basel, Switzerland). Restriction enzymes, $1 \mathrm{~Kb}$ Plus DNA ladder and T4 DNA ligase were from Invitrogen (Carlsbad, CA, USA). The jetPEI cationic polymer transfection reagent was obtained from Qbiogene (Morgan, Irvine, CA, USA). DMEM and RPMI 1640 media (both with L-glutamine), and fetal bovine serum (FBS) were obtained from PAA Laboratories GmbH (Pashing, Germany). The RetroPack PT67 retrovirus packaging cell line was purchased from BD Biosciences Clontech (Mountain View, CA, USA). The following antibodies were used: phycoerythrin (PE)-conjugated monoclonal antibody (mAb) against human TNF and an appropriate isotype control (BD Pharmingen, San Diego, CA, USA), Alexa Fluor 594-conjugated rabbit polyclonal anti-GFP IgG antibody and rabbit anti-FITC Alexa Fluor 594-conjugated polyclonal IgG antibody (used as an isotype control), purified mouse anti-

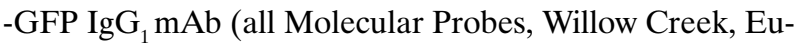
gene, OR, USA), horseradish peroxidase-conjugated goat anti-mouse IgG (Santa Cruz Biotech, Santa Cruz, CA, USA), and anti-CD14 PE-labeled mAb (BD Pharmingen). Reagents for cell fixation and permeabilization (Cytofix/ /Cytoperm and Perm/Wash) were from BD Pharmingen. The retroviral plasmid vector pMIGR1 [12] was kindly provided by Dr. Warren S. Pear (University of Pennsylvania, Philadelphia, PA, USA).

Cell cultures. The RetroPack PT67 murine dualtropic cell line was used for retrovirus packaging and production of retroviral pMIGR1 and recombinant pMIGR1-TLG (pTLG) vectors. The established human pancreatic adenocarcinoma (HPC-4) cell line [13] was used for transduction of the TLG fused gene by the recombinant retroviral infection. The PT67 cells and HPC-4 cells were grown in DMEM and RPMI 1640 media, respectively, supplemented with the following antibiotics: $50 \mu \mathrm{g} / \mathrm{ml}$ neomycin, $100 \mu \mathrm{g} / \mathrm{ml}$ ampicillin, $10 \mu \mathrm{g} / \mathrm{ml}$ tylosin, and $10 \%$ FBS referred to further as complete. Both cell lines were incubated at $37^{\circ} \mathrm{C}$ in a humidified $5 \% \mathrm{CO}_{2}$ atmosphere. All cell cultures were routinely tested for Mycoplasma $s p$. contamination using a Mycoplasma PCR ELISA kit (Roche Diagnostics GmbH, Penzberg, Germany).

Construction of fusion TLG transgene and retroviral vector preparation. The cDNA encoding human TNF (26 kDa) was obtained by the RT-PCR method using specific oligonucleotide primers and the total RNA sample isolated from human blood monocytes stimulated in vitro for $2 \mathrm{~h}$ with LPS $(1 \mu \mathrm{g} / \mathrm{ml})$. The synthesis of the first strand cDNA was carried out with enhanced avian RT and anchored oligo-dT primers according to the manufacturer's instructions. The PCR amplification of the first strand of TNF-cDNA was performed using the Pwo DNA polymerase and the following primers: BglFtnf sense 5'-GCGCAGATCTATGAGCACTGAAAGCATGAT-3' which provided BglII site (bolded) and XhoRtnf antisense 5'-CATATATCTCGAGCAGGGCAATGATCCCAA-3' which provided XhoI site (bolded). The TNF-cDNA was cleaved with BglII and XhoI restriction enzymes and ligated to the BglII-XhoI linearized vector pMIGR1 to give the recombinant pMIGR1-TNF plasmid. The LIN23-cDNA sequence coding for a linker of 23 amino acid residues was synthesized in a PCR reaction using 82-mer oligonucleotide 5'-ATATACTCGAG$\left[(\mathrm{GGC})_{4} \text { TCT }\right]_{4}$ GCCATGGTAT-3' as the template and the primers: LinSXho sense 5'-ATATACTCGAGGGCGGCGGC-3' which provided XhoI site (bolded)and LinRNco an- 
tisense 5'-GATACCATGGCAGAGCCGCC-3' which provided NcoI site (bolded). The LIN23-cDNA was cleaved with XhoI and NcoI enzymes, cloned and replaced in the pMIGR1-TNF plasmid at the XhoI-NcoI IRES fragment to give the final vector designated as PTLG which contained TLG transgene coding for the TLG fusion protein.

Production of retroviral particles. The pTLG plasmid transfer was performed using the jetPEI transfection reagent according to the manufacturer's protocol. Transfected PT67 cells expressing the TLG protein were isolated by sorting with the use of FACS Vantage with DiVa option (BD Biosciences Immunocytometry Systems, San Jose, CA, USA) equipped with an aerosol protection system (Flexoduct International, Greve, Denmark) using DiVa software. The ion laser Innova Enterprise II (Coherent, Santa Clara, CA, USA) operating at $488 \mathrm{~nm}$ was used as a light source. Sorted PT67 cells were propagated in complete DMEM medium until cell culture reached confluency and sorted again. Double-sorted PT67 cells producing retrovirus were plated at $60-80 \%$ confluency in the desired number of culture vessels. The supernatants were harvested at $24 \mathrm{~h}$ intervals until cells were no longer viable. The supernatants were collected, spun at $500 \times \mathrm{g}$ for $10 \mathrm{~min}$ and stored at $-70^{\circ} \mathrm{C}$ until use.

Transduction of human HPC-4 cells. The target HPC-4 cells were cultured in complete RPMI 1640 medium for $24 \mathrm{~h}$ at $1-2 \times 10^{5}$ per $60-\mathrm{mm}$ Petri dishes (Becton Dickinson Labware, Franklin Lakes, NJ, USA). Then the medium was replaced with $2.5 \mathrm{ml}$ of thawed supernatant from transfected PT67 cells diluted 1:1 with fresh complete RPMI 1640 medium supplemented with polybrene (final concentration $5 \mu \mathrm{g} / \mathrm{ml}$ ). The retroviral infection of the HPC- 4 cells was performed three times sequentially at $12 \mathrm{~h}$ intervals. Then the HPC- 4 cells were cultured for $48 \mathrm{~h}$ and sorted two times sequentially on the basis of the TLG protein fluorescence as described above. The purity of the sorted TLG positive cells (HPC- $4_{\text {TLG }}$ ) was checked by flow cytometry re-analysis. As the control, HPC-4 cells transduced with the original pMIGR1 retroviral vector and designated as HPC- $4_{\text {GFP }}$ were used.

Western blot analysis of TLG fusion protein expression. HPC-4 cells and their genetically modified clones, HPC$4_{\mathrm{GFP}}$ and HPC- $4_{\mathrm{TLG}}$ cells were lysed in M-PER protein extraction reagent (Pierce Biotechnol, Rochford, IL, USA) containing protease and phosphatase inhibitors (Sigma). The extracted proteins $(30 \mu \mathrm{g})$ were loaded on $4 \%$ loading gel, separated in $12 \%$ SDS gel and transferred to the polyvinylidene fluoride membrane (Immune-blot PVDF, $2 \mu \mathrm{m}$, BioRad, Hercules, CA, USA). GFP was detected with anti-GFP $\mathrm{mAb} \mathrm{IgG}_{1}$ (Molecular Probes) and with horseradish peroxidase-conjugated goat anti-mouse IgG (Santa Cruz Biotech, Santa Cruz, CA, USA), as a secondary antibody.
The membranes were developed with the SuperSignal West Pico Chemiluminescent Substrate (Pierce), dried and subsequently exposed to HyperFilm (Amersham Life Science, Little Chalfont, UK).

Intracellular detection of GFP and TLG fusion protein. Intracellular staining for the detection of GFP and TLG fusion protein was performed after cell fixation and permeabilization, as previously described [14]. Briefly, HPC-4 cells and their genetically modified clones were fixed and permeabilized with Cytofix/Cytoperm solution (BD Pharmingen) for $20 \mathrm{~min}$ at $4^{\circ} \mathrm{C}$. Then, the cells were washed twice in Perm/Wash solution (BD Pharmingen) and pelleted cells were stained $\left(30 \mathrm{~min}\right.$ at $\left.4^{\circ} \mathrm{C}\right)$ for intracellular detection of GFP and TLG fusion protein using Alexa Fluor 594-conjugated anti-GFP rabbit IgG and PE-conjugated $\mathrm{mAb}$ against human TNF. Appropriate matched isotype controls were used in parallel.

Flow cytometry analysis. Samples were analyzed in a FACS Canto flow cytometer (BD Biosciences) using DiVa software. The list mode data of 50,000 events from HPC-4 cells in a 'live gate' mode was acquired. The cells were gated according to FSC and SSC parameters. Intracellular GFP and TLG proteins were determined by green fluorescence measurement in channel FL1. Intracellular and membrane-bound TLG fusion proteins were also detected according to FL4 (Alexa Fluor 594-conjugated anti-GFP rabbit IgG) and FL2 (PE-conjugated $\mathrm{mAb}$ against human TNF) emission.

Isolation of cell populations. Human monocytes were separated from peripheral blood mononuclear cells of healthy donors by counter-flow elutriation, using a JE-6B elutriation system (Beckman Instruments, Palo Alto, CA, USA), as previously described [15]. They were $90-98 \%$ pure, as judged by staining with anti-CD14 mAb. Monocytes were suspended in RPMI 1640 medium with 5\% FBS at the concentration of $1 \times 10^{6}$ cells $/ \mathrm{ml}$ for in vitro cultures. In some experiments, monocytes isolated from a co-culture with parental or transduced HPC-4 cells (pre-exposure) at the ratio 1:0.3 for $4 \mathrm{~h}$, were used. For this purpose, after staining with PE-labeled anti-CD14 (BD Biosciences) mAb, $\mathrm{CD} 14^{+}$cells were isolated by FACS sorting (FACS Vantage with DiVa option, Becton Dickinson Immunocytometry Systems). The purity of the sorted cells was checked by flow cytometry, and exceeded $95 \%$. As control, $\mathrm{CD} 14^{+}$cells were sorted out from monocytes cultured in medium alone ('dummy sorting').

Determination of cytokine release. HPC-4, HPC- $4_{\mathrm{GFP}}$ and HPC- $4_{\text {TLG }}$ cells $\left(1 \times 10^{5} /\right.$ well $)$ were incubated in RPMI 1640 medium with 5\% FBS for $18 \mathrm{~h}$ in flat-bottom wells microtiter plates (Nunc, Roskilde, Denmark). Then, the supernatants were harvested and the concentration of TNF was 
measured by ELISA. Also, monocytes $\left(1 \times 10^{5} /\right.$ well $)$ were incubated either in the medium alone or with parental HPC-4, or HPC- $4_{\mathrm{GFP}}$ or HPC- $4_{\mathrm{TLG}}\left(3 \times 10^{4} /\right.$ well $)$ for $18 \mathrm{~h}$ (as above) and the level of TNF, IL-10 and IL-12 was determined. In some experiments, isolated $\mathrm{CD} 14^{+}$cells were cultured in the medium alone or with parental HPC-4 or their genetically modified clones (as above), and the level of cytokines was measured. All the cytokine level measurements were performed in duplicates using commercial ELISA kits (BD Pharmingen).

Cytotoxicity assay. Monocytes $\left(5 \times 10^{4} /\right.$ well $)$ were cultured for $6 \mathrm{~h}$ in the medium alone or with $\gamma$-irradiated ( $2500 \mathrm{cGy})$ HPC- 4 or HPC- $4_{\text {GFP }}$ or HPC- $4_{\text {TLG }}$ cells $\left(5 \times 10^{3} /\right.$ well $)$, and then non-irradiated HPC-4 cells $\left(2 \times 10^{4} /\right.$ well $)$ were added and cultured for a further $18 \mathrm{~h}$. The culture medium was removed and $100 \mu \mathrm{l}$ of MTT (3-(4,5-dimethylthiazol-2-yl)-2,5-diphenyltetrazolium bromide) ( $2 \mathrm{mg} / \mathrm{ml}$, Sigma) dye solution was added for the last $2 \mathrm{~h}$. Cell proliferation was assessed by the reduction of MTT. The data was expressed as the percentage of cytotoxicity calculated, as previously described [16].

Statistical analysis. The one-way analysis of variance ANOVA and post hoc Tukey test was used to calculate the statistical significance between individual treatments. Differences were considered as significant at $\mathrm{p}<0.05$.

\section{Results}

\section{TLG-transduced tumor cells}

The cDNA coding for human $26 \mathrm{kDa}$ TNF was fused to the 5' end of the cDNA coding enhanced version of the GFP protein. The short sequence of cDNA CTC GAG $\left[(\mathrm{GGC})_{4} \text { TCT }\right]_{4}$ GCC - coding for a small flexible linker was inserted between TNF-cDNA and GFP-cDNA to obtain fused gene designated as TLG.
This gene was cloned in the retroviral vector pMIGR1 [12] to obtain the final retroviral vector pTLG (Figure 1). The pTLG expression vector was used as a delivery system to HPC-4 tumor cells. The effectiveness of transduction ranged from $0.1 \%$ to $0.5 \%$. Transduced HPC-4 ${ }_{\mathrm{TLG}}$ cells following FACS sorting, yielded $1-10 \% \mathrm{TLG}^{+}$cells. After propagation of these selected cells and re-sorting, the purity of $\mathrm{TLG}^{+}$cells exceeded 95\% (Figure 2). In parallel, the HPC-4 cells mock-transduced with the original empty pMIGR1 vector were also more than $95 \% \mathrm{GFP}^{+}$(not shown). The HPC-4 ${ }_{\text {TLG }}$ cells demonstrated stable fluorescence of the TLG fusion protein, even after 30 days of maintenance in vitro in cultures with nonselective medium. The mean fluorescence intensity (MFI) of the HPC-4 ${ }_{\text {TLG }}$ cells was about 4,000 versus 200 MFI for the nontransduced cells. The percentage of TLG positive cells decreased to about $60-70 \%$

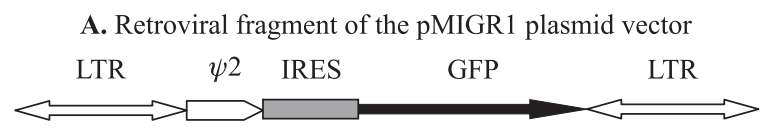

B. Retroviral fragment of the pTLG plasmid vector

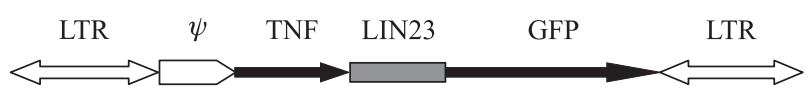

Figure 1. Linear scheme of the retroviral vector pTLG (B) construction using the parental pMIGR1 vector (A). The retroviral fragment of the vectors contain the following sequences: TNF - human pre-TNF $\alpha$-cDNA (719 bp); LTR — retroviral Long Terminal Repeats; $\psi 2$ - sequence necessary for packaging viral RNA into virus capsids; IRES - sequence Internal Rybosomal Entry Site from EMCV virus; GFP - gene coding enhanced form of Green Fluorescent Protein; LIN23 - sequence coding for linker $\left[(\mathrm{Gly})_{4} \mathrm{Ser}\right]_{4}$ Ala. The schemes of pMIGR1 and pTLG vectors do not show the prokaryotic fragment of the entire plasmid. The schemes are not drawn to scale
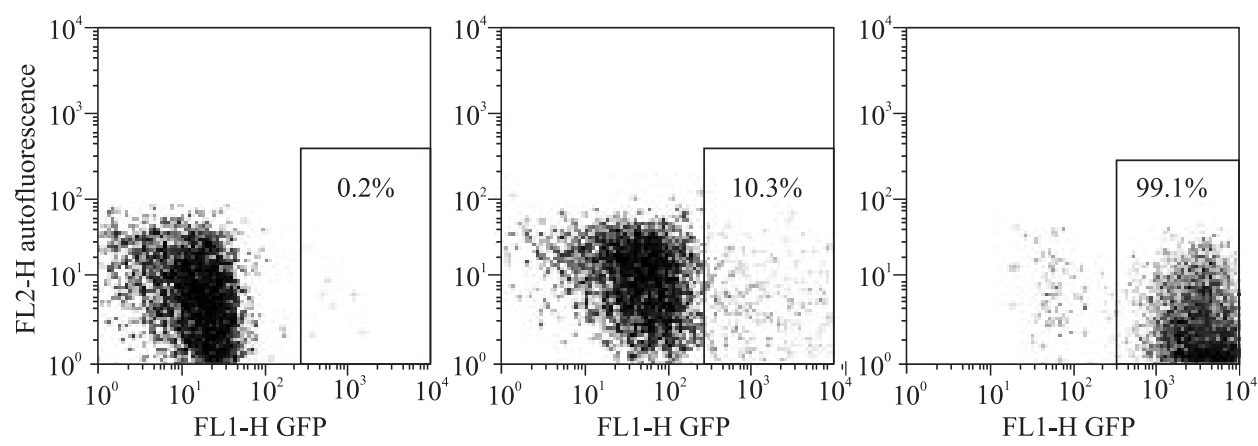

Figure 2. TLG positive HPC-4 cells detected by flow cytometry. Left dot plot - three days after transduction; middle - three days after propagation of FACS sorted initially TLG positive cells; right — three days after propagation of FACS re-sorted TLG ${ }^{+}$cells. Data from one representative experiment out of five performed is shown. The numbers show percentage of TLG positive cells in a region set according to green fluorescence (FL1) 
by day 30 post-transduction. However, the subsequent FACS sorting allowed selection of stable transductants expressing the TLG fusion protein for an additional 2-3 months in culture.

\section{TNF expression in the TLG-transduced tumor cells}

The amount of TNF secreted from HPC-4 ${ }_{\text {TLG }}$ cells was $2,428 \pm 466 \mathrm{pg} / \mathrm{ml}$ (mean $\pm \mathrm{SEM}$ ), while mock-trans-

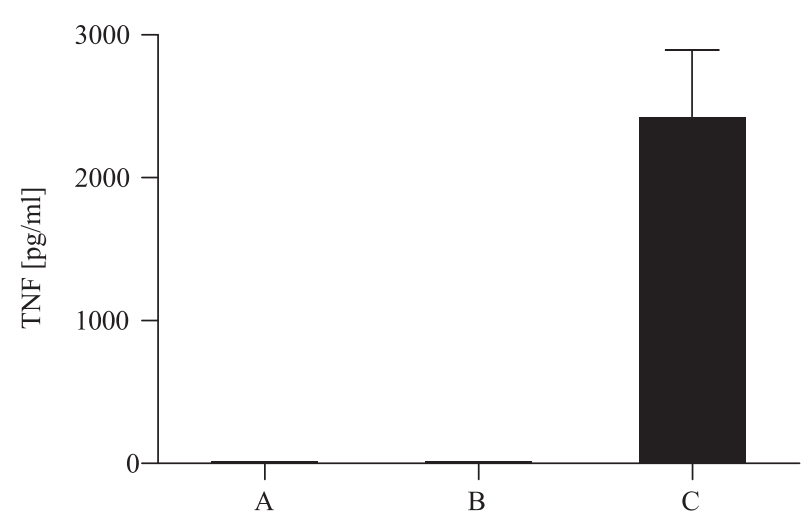

Figure 3. TNF secretion by: (A) HPC-4, (B) HPC-4 $4_{\text {GFP }}$ and (C) HPC-4 ${ }_{\text {TLG }}$ cells. Data is based on five independent experiments, two replicates per treatment, and expressed as $\mathrm{pg} / \mathrm{ml} / 1 \times 10^{5}$ cells $/ 18$ hours. Mean values $\pm \mathrm{SEM}$ are shown; HPC- $4_{\text {TLG }}$ vs. HPC-4 and HPC- $4_{\text {GFP }}$ p $<0.001$ duced HPC- $4_{\text {GFP }}$ and parental HPC-4 cells did not produce TNF (Figure 3 ). No IL-6, IL-10, MIP1 $\alpha$ or RANTES were found in the supernatants of HPC$-4_{\text {TLG }}$ cells (data not shown).

As membrane bound TNF is regarded as important in the cellular interactions [17], in the next step we analyzed whether synthesized TLG fusion protein was expressed as the membrane-bound form. For this purpose, transduced HPC-4 ${ }_{\text {TLG }}$ cells were stained with anti-TNF PE-conjugated $\mathrm{mAb}$. However, taking into consideration significant spillover of green fluorescence from enhanced GFP into PE channel (data not shown), we took an advantage from anti-GFP antibodies conjugated with Alexa Fluor 594, the dye which did not interfere with PE emission spectrum. Anti-GFP antibodies were used in parallel with anti-TNF mAb for cell staining. After staining, the cells were analyzed by flow cytometry according to FL4 (anti-GFP) and FL2 (anti-TNF) fluorescence. In comparison to control HPC-4 and HPC- $4_{\text {GFP }}$ cells, two-color flow cytometry analysis of HPC- $4_{\text {TLG }}$ cells showed that $30.9 \%$ of cells stained with anti-TNF $\mathrm{mAb}, 31.3 \%$ were $\mathrm{GFP}^{+}$while $19.5 \%$ were both TNF and GFP positive (Figure 4). Intracellular staining of HPC- $4_{\text {TLG }}$ cells with anti-TNF and anti-GFP Abs showed 40.6\% $\mathrm{TNF}^{+}$and $\mathrm{GFP}^{+}$95.3\% cells (Figure 5A). Anti-GFP Ab stained $84.3 \%$ of HPC- $4_{\text {GFP }}$ cells and none of parental HPC-4 cells.
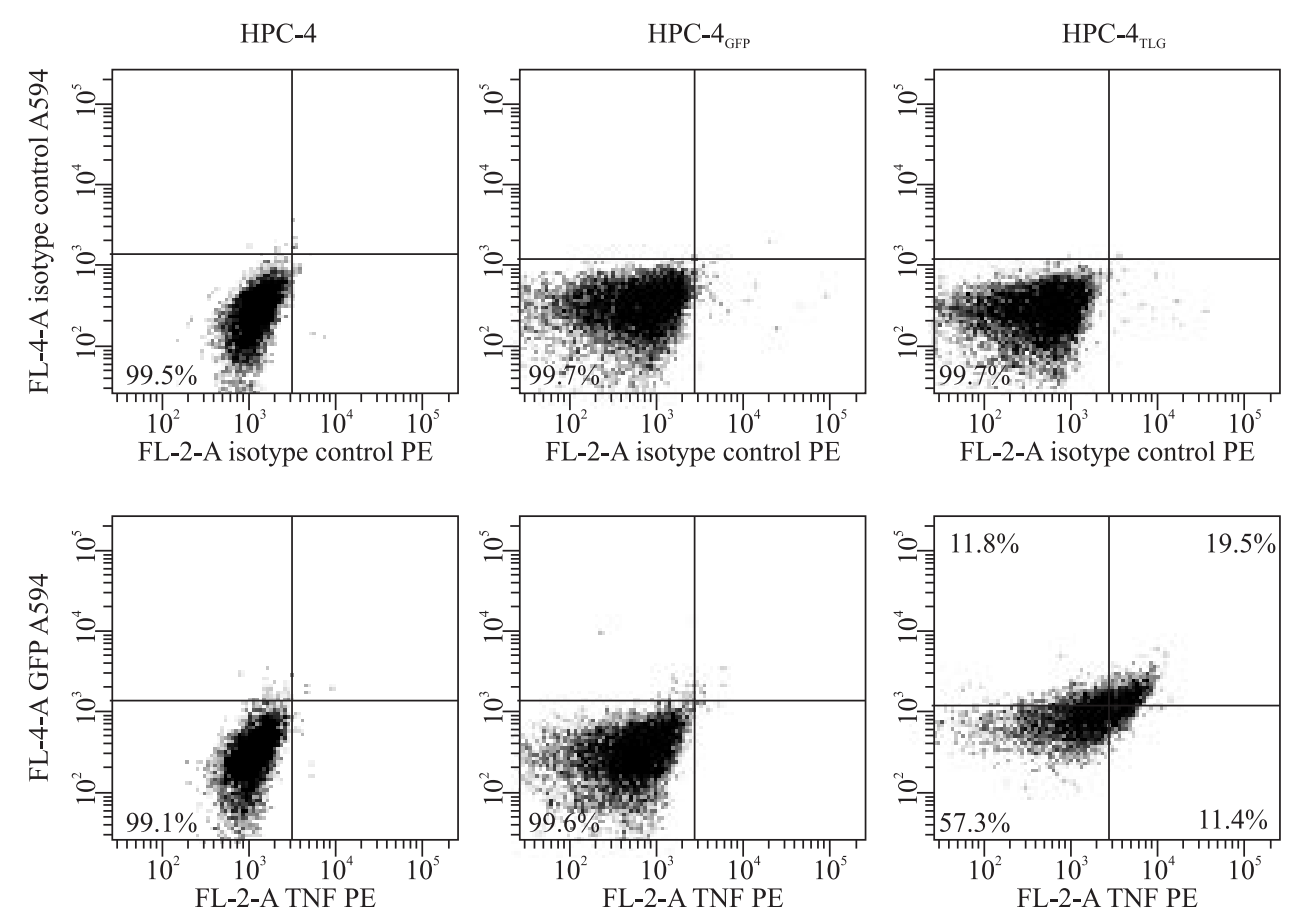

Figure 4. Expression of membrane-bound TLG fusion protein by HPC-4, HPC-4 ${ }_{\mathrm{GFP}}$ and HPC-4 ${ }_{\mathrm{TLG}}$ cells. Cells were stained with PE-conjugated anti-TNF mAb and anti-GFP Alexa Fluor-594 conjugated Ab, and were analyzed by flow cytometry according to FL2 (PE) vs. FL4 (Alexa Fluor-594) fluorescence. Representative data from one out of four experiments performed is shown 

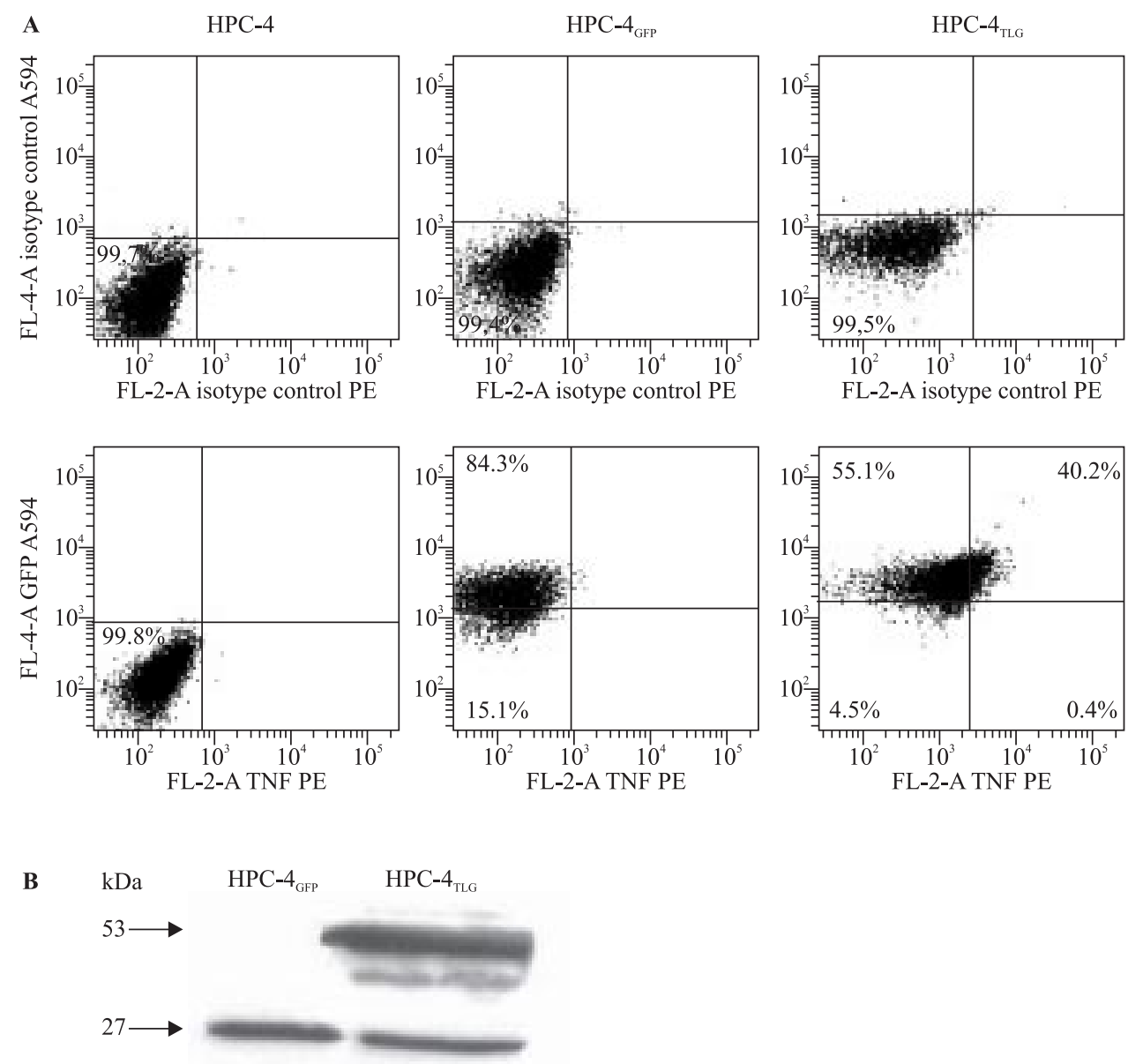

Figure 5. Expression of intracellular TLG fusion protein by: (A) flow cytometry and (B) Western blot analysis.

A. HPC-4, HPC- $4_{\text {GFP }}$ and HPC- $4_{\mathrm{TLG}}$ cells. Cells were fixed and after permeabilization were stained for intracellular expression of TNF and GFP, as in Figure 4. Cells were analyzed by two-color flow cytometry according to FL2 vs. FL4 fluorescence. Representative data from one out of four experiments performed is shown. B. Western blot analysis of intracellular expression and localization of GFP protein $(27 \mathrm{kDa})$ and TLG fusion protein $(53 \mathrm{kDa})$ in the lysates of transduced HPC-4 ${ }_{\text {GFP }}$ and HPC-4 ${ }_{\text {TLG }}$ cells using anti-GFP IgG $\mathrm{mAb}_{1}$ as the primary and horseradish peroxidase-conjugated goat anti-mouse IgG as the secondary Abs

The intracellular co-expression of TNF and GFP in HPC- $4_{\text {TLG }}$ cells was confirmed by Western blot analysis of lysates from TLG transduced cancer cells (Figure 5B).

\section{Induction of cytokines in monocytes stimulated with TLG-transduced tumor cells}

Monocytes were cultured for $18 \mathrm{~h}$ with $\mathrm{HPC}-4_{\mathrm{TLG}}$ or HPC-4 $4_{\text {GFP }}$ or parental HPC-4 cells to test whether they stimulated differently TNF, IL-10 and IL-12 production in monocytes. In comparison to parental HPC-4, HPC $-4_{\text {TLG }}$ cells induced significantly higher release of TNF, while the induction of IL-10 was significantly lower, and no IL-12 was detected (Figure 6). Interestingly, monocytes stimulated with mock-transduced HPC- $4_{\text {GFP }}$ cells produced significantly less TNF and no IL-10 or IL-12 compared to those stimulated with parental HPC-4 cells.

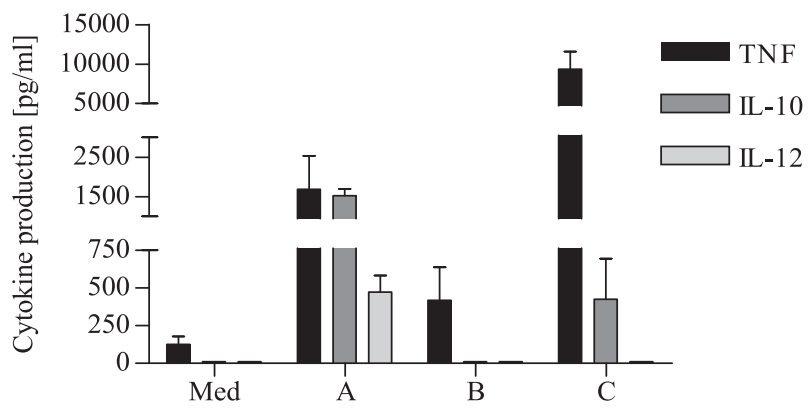

Figure 6. Secretion of TNF, IL-10 and IL-12 by monocytes cultured in the medium (Med) or with: (A) HPC-4 or (B) HPC- $4_{\text {GFP }}$ or (C) HPC- $4_{\text {TLG }}$ cells for 18 h. No cytokines were released by tumor cells except HPC- $4_{\mathrm{TLG}}$, which secreted $900 \pm 180 \mathrm{pg} / \mathrm{ml}$ of $\mathrm{TNF} / 3 \times 10^{4} \mathrm{cells} / \mathrm{ml}$ (not shown).

The results of five different experiments, two replicates per treatment (mean \pm SEM) are shown; for TNF: HPC-4 vs. HPC- $4_{\text {TLG }} \mathrm{p}<0.01$, HPC- $4_{\text {GFP }}$ vs. HPC- $4_{\text {TLG }} \mathrm{p}<0.01$; for IL-10: HPC-4 vs. HPC-4 $4_{\text {TLG }}$ p $<0.01$, HPC-4 vs. HPC-4 $4_{\text {GFP }}$ $\mathrm{p}<0.001$; for IL-12: HPC-4 vs. HPC-4 ${ }_{\text {GFP }}$ and HPC- $4_{\text {TLG }} \mathrm{p}<0.01$ 


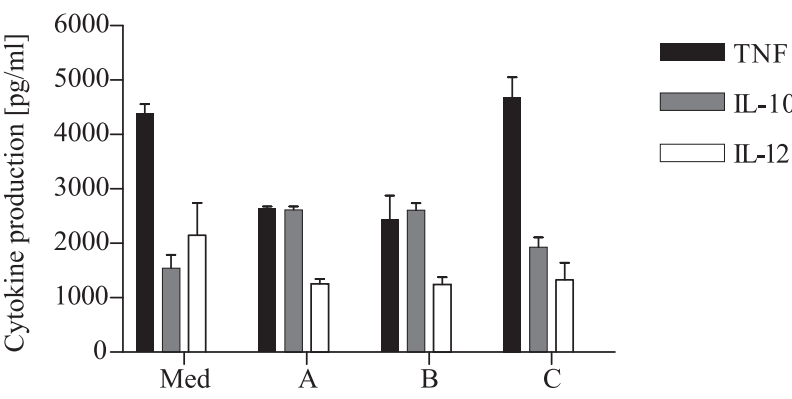

Figure 7. Secretion of TNF, IL-10 and IL-12 by monocytes pre-exposed to tumor cells. Monocytes were pre-incubated in the medium alone (Med) or with (A) HPC-4 or (B) $\mathrm{HPC}-4_{\text {GFP }}$ or (C) HPC-4 ${ }_{\mathrm{TLG}}$ cells for $4 \mathrm{~h}$. Then CD14 monocytes were isolated by FACS sorting and stimulated with HPC-4 cells. The results of four different experiments, two replicates per treatment (mean \pm SEM) are shown; HPC-4 vs. HPC- $4_{\text {TLG }} \mathrm{p}<0.05$, HPC- $4_{\text {GFP }}$ vs. HPC-4 $4_{\text {TLG }}$ $\mathrm{p}<0.05$ (for TNF and IL-10 only)

\section{Transduced tumor cells prevent deactivation of monocytes}

We have previously shown that short pre-exposure of monocytes to tumor cells deactivated them, as evidenced by decreased TNF and enhanced IL-10 production [11]. In order to check whether transduced HPC-4 cells act similarly, monocytes were cultured for $4 \mathrm{~h}$ with parental HPC-4 or transduced HPC- $4_{\text {GFP }}$ or $\mathrm{HPC}-4_{\mathrm{TLG}}$ cells. CD $14^{+}$cells were then isolated by FACS sorting and stimulated with HPC-4 cells for $18 \mathrm{~h}$. Figure 7 shows that pre-exposure of monocytes to parental HPC-4 cells inhibited TNF and IL-12 and enhanced IL-10 production. In contrast, $\mathrm{HPC}-4_{\mathrm{TLG}}$ cells neither caused inhibition of TNF and IL-12 synthesis nor enhancement of IL-10 production. Pre-exposure of monocytes to mock-transduced HPC- $4_{\text {GFP }}$ cells caused similar production of TNF, IL-10 and IL-12 as those pre-exposed to parental cells.

\section{Cytotoxicity of monocytes pre-exposed to $\gamma$-irradiated HPC-4 ${ }_{T L G}$}

Monocytes/macrophages possess significant spontaneous cytotoxicity against tumor cells [16], which is decreased by the pre-exposure to tumor cells. This decrease is a part of the monocytes' deactivation. To check whether HPC- $4_{\text {TLG }}$ cells may also prevent inhibition of cytotoxicity, monocytes were preincubated with $\gamma$-irradiated HPC- 4 or HPC- $4_{\mathrm{GFP}}$, or HPC- $4_{\mathrm{TLG}}$ cells, and cytotoxicity against HPC-4 tumor cells was measured. As expected, monocytes pre-exposed to HPC-4 or HPC- $4_{\text {GFP }}$ cells demonstrated lowered cytotoxicity (Figure 8). In contrast, there was no inhibition of cytotoxicity when monocytes were pre-exposed to $\mathrm{HPC}-4_{\mathrm{TLG}}$ cells.

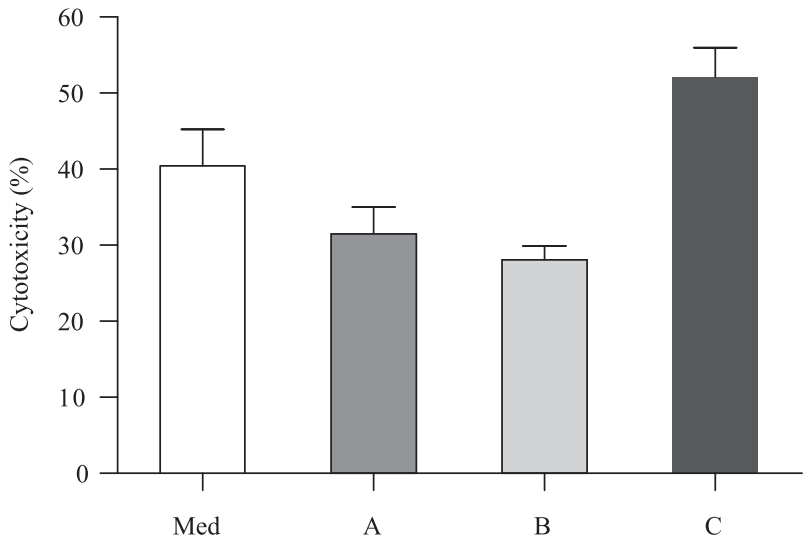

Figure 8. Cytotoxic activity against HPC-4 cells of monocytes incubated in the medium alone (Med - control), preexposed to $\gamma$-irradiated: (A) HPC-4 or (B) HPC- $4_{\text {GFP }}$ or (C) HPC- $4_{\text {TLG }}$ cells. Cell proliferation was assessed by MTT reduction. Data (mean \pm SEM) based on four experiments, two replicates per treatment, expressed as the percentage of cytotoxicity is shown; HPC-4 vs. HPC-4 $4_{\text {TLG }}$ $\mathrm{p}<0.05 ; \mathrm{HPC}^{-} 4_{\text {GFP }}$ vs. HPC- $4_{\text {TLG }} \mathrm{p}<0.05$

\section{Discussion}

For a successful cancer gene therapy, it is fundamental to obtain as many cells transduced with the therapeutic gene as possible. Thus, knowing the actual gene transfer rate is critical. The GFP has made a breakthrough in gene transfer technology. It is a small protein that fits most of the currently used vectors and can be fused with other proteins to yield therapeutic fusion proteins $[5,18,19]$. The use of GFP fusion proteins provides enhanced sensitivity and resolution compared to antibody staining techniques and permits kinetic studies of a fusion protein localization $[4,20]$. The construction of the fusion protein often requires the linking of two proteins or protein domains by an appropriate polypeptide linker. Many studies have suggested that the flexibility and hydrophilicity of the linker were very important in preventing the disturbance of the functions of both domains [21, 22].

In the present study, the TLG fusion gene was constructed by joining the TNF-cDNA and enhanced GFP-cDNA with the LIN23-DNA, coding for a short, flexible and hydrophilic linker Leu Glu $\left[(\mathrm{Gly})_{4} \mathrm{Ser}_{4}\right.$ Ala, which allowed retention of GFP and TNF expression. The retroviral vector pTLG carrying the TLG fusion gene was used for transduction of HPC-4 cells. The TLG gene transduced cells exhibited unchanged morphology and proliferation during in vitro culture for more than six months as compared to the parental nontransduced cells. The TLG gene transduced cells secreted a substantial amount of TNF, the presence of which was observed on their mem- 
brane and intracellularly. One could argue that only a part of the $\mathrm{GFP}^{+}$cells express TNF at the same time (Figures 4 and 5). In the preliminary set of experiments, we compared membrane and intracellular expression of TNF in HPC-4 ${ }_{\mathrm{TLG}}$ cells without anti-GFP staining. Under such conditions, the amount of double positive cells was only around $50-60 \%$ of $\mathrm{GFP}^{+}$ cells (for both membrane and intracellular staining - data not shown). Such a relatively small amount of double positive cells could be a result of a spillover of green fluorescence from enhanced GFP to the PE channel, which could not be fully compensated during FACS analysis. To resolve this issue, we changed our detection method and introduced staining with anti-GFP Ab conjugated with Alexa Fluor 594, a dye which does not interfere with PE. This approach, however, did not improve the expression ratio, neither in surface nor intracellular staining. Thus, the relatively low number of double positive HPC- $4_{\text {TLG }}$ cells during membrane staining was probably due to secretion of TNF, cleaved rapidly from the TLG protein, to the medium. In the case of intracellular staining, the high amount of TLG present in the cytoplasm is probably neither properly folded nor fully accessible for both antibodies used for FACS analysis. Such an explanation is supported by Western blot analysis of HPC$-4_{T L G}$ cell lysates showing intracellular expression of the fusion protein (Figure 5B). It shows that there is also a band of free GFP present, which was probably left after the cleavage of TNF from TLG and its secretion. However, the exact trafficking and processing of the TLG fusion protein in transduced HPC-4 cancer cells has not been investigated in the present paper, and should be addressed in our future studies.

It has been previously shown that monocytes respond to tumor, but not normal, cells by producing several toxic mediators: TNF, ROI, radical nitrogen intermediates (RNI) and increased cytotoxicity [16, $23,24]$. On the other hand, clinicopathological evidence suggests that TIM, of which monocytes are precursors, may enhance the growth of some tumors [25]. Our previous data showed that although the first contact of monocytes with tumor cells induces antitumor activity, secondary contact leads to their deactivation (diminished TNF, IL-12 and ROI production, decreased cytotoxicity and enhanced IL-10 production), i.e. they became M2 polarized monocytes [25].

In the present experiments, we have investigated how the contact with tumor cells expressing TNF changes the activity of monocytes. In comparison to parental cells, HPC-4 ${ }_{\text {TLG }}$ cells induced higher production of TNF by monocytes and lower IL-10. Contrary to what could be expected, we found that HPC- $4_{\mathrm{GFP}}$ cells, compared to parental HPC-4 cells, caused a decrease in the secretion of all tested cytokines by monocytes. Although we have no formal proof, this phenomenon may be due to more accelerated apoptosis of GFP transduced cells [26], which are rapidly phagocytosed by monocytes during their co-culture. Phagocytosis of apoptotic cells by monocytes, as evidenced by us and others, does not lead to secretion of TNF, IL-10 or IL-12. The only cytokine released by monocytes/macrophages during phagocytosis of apoptotic cells is $\operatorname{TGF} \beta$, which was not analyzed in our study. Another concern may arise when analyzing TNF secretion in the co-culture of monocytes and HPC- $4_{\text {TLG }}$ cells. In this case, the question is whether the TNF release comes from the transduced cells (GFP-TNF), or from the monocytes alone. Indeed, in this situation both cell populations may be a source of TNF; however, as indicated in the legend of Figure 6 , the amount of TNF secreted by HPC- $4_{\text {TLG }}$ cells $\left(900 \pm 180 \mathrm{pg} / \mathrm{ml}\right.$ of TNF $/ 3 \times 10^{4}$ cells $\left./ \mathrm{ml}\right)$ is far less compared to the level secreted by monocytes, thus having little effect on the total level of this cytokine in the co-culture supernatants.

The $\mathrm{CD} 14^{+}$monocytes sorted out from the co-culture with HPC-4 cells and restimulated with HPC-4, showed decreased TNF and IL-12 production. However, $\mathrm{CD} 14^{+}$cells isolated from the co-culture with HPC- $4_{\text {TLG }}$ produced a higher level of TNF and IL-12 and a diminished level of IL-10. This may suggest that, in the presence of TNF on the cell surface of HPC-4 TLG or released by them, no deactivation of monocytes occurred. The observation that cytotoxic activity of monocytes pre-exposed to HPC-4 ${ }_{\text {TLG }}$ cells was not diminished also confirmed this suggestion.

The ability of TNF to activate monocytes/macrophages has been widely reported [17, 27, 28]. Activated monocytes/macrophages produce several toxic mediators such as RNI, ROI and TNF, which are probably directly involved in tumor cell killing in vitro. TNF may be also involved in indirect tumor cell killing by inducing RNI and ROI [29]. The inhibition of tumor growth after transduction of cancer cells with DNA construct coding for TNF has been observed, although different experimental models and divergent results concerning the activity of membrane-bound vs. secreted TNF have been reported $[6,17,30]$. It has been suggested that in immunocompetent mice, tumor regression was $\mathrm{T}$ cell-dependent $[6,30]$, while in immunodeficient mice the effect was macrophage-dependent [6]. Membrane bound TNF is regarded as important in the cellular interactions, e.g. with murine macrophages [17]. In keeping with this, HPC-4 cells effectively stimulated human monocytes for TNF release, while decreasing IL-10 and IL-12 secretion.

In summary, the present data indicates that human cancer cells transduced with TNF-cDNA release TNF and express its membrane-bound form. These 
cells induced a higher TNF production by monocytes, and prevented their deactivation, thus giving a rationale for tumor cell based immunotherapy of cancer.

\section{Acknowledgment}

This study was supported by Jagiellonian University Medical College Grant no. 501/NKL/112/L.

\section{References}

1. Ausubel FM, Brent R, Kingdom RE et al. Current Protocols in Molecular Biology. John Wiley \& Sons, NY; 1996; Suppl., 36, unit 9.11:1-18.

2. Maxfield LF, Fraize CD, Coffin JM. Relationship between retroviral DNA-integration-site selection and host cell transcription. Proc Natl Acad Sci USA. 2005;102:1436-1441.

3. Weidhaas JB, Angelichio EL, Fenner S, Coffin JM. Relationship between retroviral DNA integration and gene expression. $J$ Virol. 2000;74:8382-8389.

4. Gerdes HH and Kaether C. Green fluorescent protein: applications in cell biology. FEBS Lett. 1996;389:44-47.

5. Gubin AN, Koduru S, Njoroge JM, Bhatnagar R, Miller JL. Stable expression of green fluorescent protein after liposomal transfection of K562 cells without selective growth conditions. Biotechniques. 1999;27:1162-1164,1166-1170.

6. Asher AL, Mule JJ, Kasid A et al. Murine tumor cells transduced with the gene for tumor necrosis factor-alpha. Evidence for paracrine immune effects of tumor necrosis factor against tumors. J Immunol. 1991;146:3227-3234.

7. Blankenstein T, Quin Z, Uberla K et al. Tumor suppression after tumor cell-targeted tumor necrosis factor alpha gene transfer. J Exp Med. 1991;173:1047-1052.

8. Danoff G, Jaffee E, Lazenby A et al. Vaccination with irradiated tumor cells engineered to secrete murine granulocytemacrophage colony-stimulating factor stimulates potent, specific, and long-lasting anti-tumor immunity. Proc Natl Acad Sci USA. 1993;90:3539-3543.

9. Gattinoni L, Powell Jr. DJ, Resenberg SA, Restifo NP. Adoptive immunotherapy for cancer: building on success. Nature Rev Immunol. 2006;6:383-393.

10. Lewis CE, Pollard JW. Distinct role of macrophages in different tumor microenvironments. Cancer Res. 2006;66:605-612.

11. Mytar B, Wołoszyn M, Szatanek R et al. Tumor cell-induced deactivation of human monocytes. $J$ Leukocyte Biol. 2003; 74:1094-1101.

12. Pear WS, Miller JP, Xu L et al. Efficient and rapid induction of a chronic myelogenous leukemia-like myeloproliferative disease in mice receiving P210 bcr/abl-transduced bone marrow. Blood. 1998;92:3780-3792.

13. Mytar B, Siedlar M, Wołoszyn M, Colizzi V, Zembala M. Cross-talk between human monocytes and cancer cells during reactive oxygen intermediates generation: the essential role of hyaluronan. Int J Cancer. 2001;94:727-732.

14. Baran J, Kowalczyk D, Ożóg M, Zembala M. Three colour flow cytometry detection of intracellular cytokines in peripheral blood mononuclear cells: comparative analysis of phor- bol myristate acetate-ionomycin and phytohemagglutinin stimulation. Clin Diag Lab Immunol. 2001;8:303-313.

15. Baran J, Guzik K, Hryniewicz W, Ernst M, Flad H-D, Pryjma $\mathrm{J}$. Apoptosis of monocytes and prolonged survival of granulocytes as a result of phagocytosis of bacteria. Infect Immun. 1996;64:4242-4248.

16. Mytar B, Siedlar M, Wołoszyn M, Ruggiero I, Pryjma J, Zembala $\mathrm{M}$. Induction of reactive oxygen intermediates in human monocytes by tumour cells and their role in spontaneous monocyte cytotoxicity. Br J Cancer. 1999;79:737-743.

17. Nagy T, Glavinas H, Szincsak $N$ et al. Tumor cells expressing membrane-bound tumor necrosis factor activate macrophages and have a compromised growth in immunosuppressed and immunodeficient mice. Cancer Lett. 2003;196:49-56.

18. Bennett RP, Cox CA, Hoeffler JP. Fusion of green fluorescent protein with the Zeocin-resistance marker allows visual screening and drug selection of transfected eukaryotic cells. Biotechniques. 1998;24:478-482.

19. Oh SC, Nam SY, Kwon HC, Kim CM et al. Generation of fusion genes carrying drug resistance, green fluorescent protein, and herpes simplex virus thymidine kinase genes in a single cistron. Mol Cells. 2001;11:192-197.

20. Hoffman RM. Visualization of GFP-expressing tumors and metastasis in vivo. Biotechniques. 2001;30:1016-1026.

21. Arai R, Ueda H, Kitayama A, Kamiya N, Nagamune T. Design of the linkers which effectively separate domains of a bifunctional fusion protein. Protein Eng. 2001;14:529-532.

22. Michael NP, Chester KA, Melton RG et al. In vitro and in vivo characterisation of a recombinant carboxypeptidase G2: anti-CEA scFv fusion protein. Immunotechnology. 1996;2:47-57.

23. Zembala M, Czupryna A, Więckiewicz J et al. Tumour-cell-induced production of tumour necrosis factor by monocytes of gastric cancer patients receiving BCG immunotherapy. Cancer Immunol Immunother. 1993;36:127-132.

24. Zembala M, Siedlar M, Marcinkiewicz J, Pryjma J. Human monocytes are stimulated for nitric oxide release in vitro by some tumor cells but not by cytokines and lipopolysaccharide. Eur J Immunol. 1994;24:435-439.

25. Allavena P, Sica A, Solinas G, Porta C, Montovani A. The inflammatory micro-environment in tumour progression: the role of tumour-associated macrophages. Crit Rev Oncol Hematol. 2008;66:1-9.

26. Liu H-S, Jan M-S, Chou C-K, Chen P-H, Ke N-J. Is green fluorescein protein toxic to the living cells? Biochem Biophys Res Commun. 1999;260:712-717.

27. Sethu S, Mendez-Corao G, Melendez AJ. Phospholipase D1 plays a key role in TNF-alpha signaling. J Immunol. 2008;180: 6027-6034.

28. Talmadge JE, Phillips H, Schneider M et al. Immunomodulatory properties of recombinant murine and human tumor necrosis factor. Cancer Res. 1988;48:544-550.

29. Klimp AH, de Vries EG, Scherphof GL, Daemen T. A potential role of macrophage activation in the treatment of cancer. Crit Rev Oncol Hematol. 2002;44:143-161.

30. Karp SE, Hwu P, Farber A et al. In vivo activity of tumor necrosis factor (TNF) mutants. Secretory but not membranebound TNF mediates the regression of retrovirally transduced murine tumor. J Immunol. 1992;149:2076-2081.

Submitted: 20 April, 2010

Accepted after reviews: 30 January, 2011 